\title{
Association of assisted reproductive technology (ART) treatment and parental infertility diagnosis with autism in ART-conceived children
}

\author{
D.M. Kissin ',*, Y. Zhang ', S.L. Boulet ', C. Fountain ', P. Bearman 3 , \\ L. Schieve ${ }^{4}$, M. Yeargin-Allsopp ${ }^{4}$, and D.J. Jamieson ${ }^{1}$
}

\begin{abstract}
'National Center for Chronic Disease Prevention and Health Promotion, Centers for Disease Control and Prevention, Atlanta, GA, USA ${ }^{2}$ Department of Sociology and Anthropology, Fordham University, New York, NY, USA ${ }^{3}$ Interdisciplinary Center for Innovative Theory and Empirics, Columbia University, New York, NY, USA ${ }^{4}$ National Center on Birth Defects and Developmental Disabilities, Centers for Disease
\end{abstract} Control and Prevention, Atlanta, GA, USA

*Correspondence address. Centers for Disease Control and Prevention, Division of Reproductive Health, 4770 Buford Highway NE, Mailstop F-74, Atlanta, GA 3034I, USA. Tel: + I-770-488-6408; E-mail: dkissin@cdc.gov

Submitted on June 2, 20I 4; resubmitted on October 29, 20I 4; accepted on November 27, 2014

STUDY QUESTION: Are assisted reproductive technology (ART) treatment factors or infertility diagnoses associated with autism among ART-conceived children?

SUMMARY ANSWER: Our study suggests that the incidence of autism diagnosis in ART-conceived children during the first 5 years of life was higher when intracytoplasmic sperm injection (ICSI) was used compared with conventional IVF, and lower when parents had unexplained infertility (among singletons) or tubal factor infertility (among multiples) compared with other types of infertility.

WHAT IS KNOWN ALREADY: Some studies found an increased risk of autism among ART-conceived infants compared with spontaneouslyconceived infants. However, few studies, and none in the USA, have examined the associations between types of ART procedures and parental infertility diagnoses with autism among ART-conceived children.

STUDY DESIGN, SIZE, DURATION: Population-based retrospective cohort study using linkages between National ART Surveillance System (NASS) data for 1996-2006, California Birth Certificate data for 1997-2006, and California Department of Developmental Services (DDS) Autism Caseload data for 1997-2011.

PARTICIPANTS/MATERIALS, SETTING, METHODS: All live born ART-conceived infants born in California in 1997-2006 ( $n=42383$ ) with 5-year observation period were included in the study. We assessed the annual incidence of autism diagnosis documented in DDS, which includes information on the vast majority of persons with autism in California, and the association of autism diagnosis with ART treatment factors and infertility diagnoses.

MAIN RESULTS AND THE ROLE OF CHANCE: Among ART-conceived singletons born in California between 1997 and 2006 , the incidence of autism diagnosis remained at $\sim 0.8 \%$ ( $P$ for trend 0.19 ) and was lower with parental diagnosis of unexplained infertility (adjusted hazard risk ratio [aHRR]; 95\% confidence interval: 0.38; 0.15-0.94) and higher when ICSI was used (aHRR I.65; I.08-2.52), when compared with cases without these patient and treatment characteristics. Among ART-conceived multiples, the incidence of autism diagnosis between 1997 and 2006 remained at $\sim 1.2 \%$ ( $P$ for trend 0.93 ) and was lower with parental diagnosis of tubal factor infertility (aHRR 0.56; $0.35-0.90)$ and higher when ICSI was used (aHRR I.7I; I.I0-2.66).

LIMITATIONS, REASONS FOR CAUTION: Study limitations include imperfect data linkages, lack of data on embryo quality and possible underestimation of autism diagnosis cases. Limitations of the observational study design could affect the analysis by the possibility of residual confounders. Since information about ICSI use was missing for most frozen/thawed embryo transfer cycles, our findings of association of ICSI use and autism diagnosis can only be generalizable to fresh embryo transfer cycles.

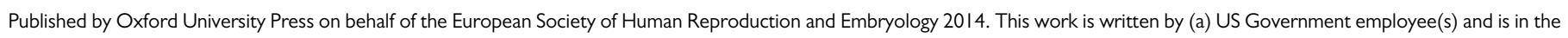
public domain in the US. 
WIDER IMPLICATIONS OF THE FINDINGS: Our study provides additional evidence of the association between some types of ART procedures with autism diagnosis. Additional research is required to explain the increased risk of autism diagnosis with ICSI use, as well as studies on the effectiveness and safety of ICSI.

STUDY FUNDING/COMPETING INTEREST(S): The study was partially supported by the National Institutes of Health. The authors have no competing interests that may be relevant to the study.

Key words: assisted reproductive techniques / IVF / infertility / ICSI / autistic disorder

\section{Introduction}

The use of assisted reproductive technology (ART), which includes all procedures that involve handling of oocytes and sperm or embryos outside of the human body, has been increasing steadily since it was first introduced in 1978. Over 5 million children conceived with ART have been born globally (Adamson et al., 20I3). About I.5\% of all infants born in the USA are conceived using ART (Sunderam et al., 20I3). The effect of ART procedures on the health and development of children has been an area of special interest for researchers during the last three decades. However, studying the long-term outcomes of ART is difficult in part due to rapid technological progress in this relatively new field of medicine. Remarkable advances in embryo culture, cryopreservation of embryos and oocytes, ICSI, preimplantation genetic testing, and assisted hatching have led to the development of new treatment options. The safety-including long-term safety — of these new treatments requires careful study.

One area of interest is the potential association between ART fertility treatments and autism, a serious neurodevelopmental disorder characterized by impairments in social functioning and communication, and repetitive, stereotyped behaviors, which commonly co-occurs with intellectual disability (CDC, 20I4). The prevalence of identified autism rapidly increased during the last decade (CDC, 20I4). Results of studies that have compared the risk of autism among ART-conceived and spontaneously-conceived infants are mixed (Stein et al., 2006; Maimburg and Vaeth, 2007; Hvidtjorn et al., 2009, 20II; Zachor and Ben Itzchak, 20 I I; Lyall et al., 2012; Conti et al., 2013; Hart and Norman, 2013; Lehti et al., 2013). It is uncertain whether positive associations are accounted for by the ART procedure itself or underlying characteristics of ART patients and increased incidence of multiple births after ART. Few studies, and none in the USA, have examined the associations between types of ART procedures and parental infertility diagnoses with autism among ART-conceived children (Hvidtjorn et al., 20 I I; Bay et al., 20 I3; Sandin et al., 20 I3). A recent study from Sweden found that fresh embryo transfer ART procedures using ICSI for male factor infertility were associated with increased risk of autistic disorder and intellectual disability in offspring compared with fresh embryo transfer procedures without ICSI (Sandin et al., 20I3). However, a study from Denmark found no effect of ICSI or conventional IVF on neurodevelopment of children (Bay et al., 20I3).

In the USA, the use of ICSI, originally developed for the treatment of male factor infertility (Palermo et al., 1992), has increased dramatically during the last decade and is now used in $67 \%$ of all ART procedures regardless of infertility diagnosis (CDC, 20I3a). Our study objectives were to assess whether ART treatment factors or infertility diagnoses among couples who use ART are associated with autism. Based on the results from previous studies, we specifically investigated the association of ICSI use with autism while controlling for other treatment factors.

\section{Methods}

\section{Study population and data sources}

This population-based retrospective cohort study included all live born ARTconceived infants born in 1997-2006 in the state of California. The 2006 cutoff was chosen to ensure that each child in the study has fixed 5-year observation period. The study is based on linkages between National ART Surveillance System (NASS) data for 1996-2006, California Birth Certificate data for 1997-2006 and California Department of Developmental Services (DDS) Autism Caseload data for 1997-20II. NASS data were linked with California Birth Certificate data using LinkPlus 2.0 linkage software (CDC, Atlanta, Georgia) (Zhang et al., 20I2). Due to the absence of direct identifiers in NASS, the probabilistic linkage method utilized the following indirect identifiers: maternal date of birth, infant's date of birth, plurality, zip code and gravidity. Uncertain or duplicate matches were reviewed and resolved manually by using additional variables: infant sex, maternal race and infant birthweight. Average linkage rate for all years was $90 \%$ (ranging from 88 to 93\%). Similar linkages of NASS and Birth Certificate data in other states were conducted as part of States Monitoring ART (SMART) Collaborative; validation of such linkages showed high sensitivity and specificity (Mneimneh et al., 20I3). California Birth Certificate data were linked with California DDS Autism Caseload data using a combination of deterministic and probabilistic linkage methods with the following direct and indirect identifiers: child's name, date of birth, sex, race/ethnicity and zip code. Uncertain or duplicate matches were reviewed and resolved manually using additional variables. Average linkage rate was $86 \%$ (ranging from 82 to $89 \%$ ). Although more data points were available to link California Birth Certificate data with California DDS Autism Caseload data, migration in and out of the state during the time between birth and autism diagnosis (up to 5 years) could result in lower linkage rates.

\section{Exposure (ART and related variables)}

The National ART Surveillance System (NASS) is maintained by the Division of Reproductive Health, Centers for Disease Control and Prevention (CDC). Fertility clinics in the USA are mandated to report data on each ART cycle to the CDC by the Fertility Clinic Success Rates and Certification Act of 1992 (FCSRCA, 1992). NASS covers an estimated $97 \%$ of all ART cycles performed in the USA (CDC, 20I3a). Data abstracted from medical records include patients' demographics, obstetric history, detailed information on the ART cycle and information on cycle outcome. The latter is obtained by active follow-up by fertility clinic and includes information about whether the cycle resulted in pregnancy, pregnancy outcome and information about each resultant neonate (gestational age, birthweight and sex). The data from each clinic are validated by the clinics' medical director prior to submission. In addition, $\sim 7-10 \%$ of reporting clinics are randomly selected each year for validation by the CDC, during which certain data elements reported by the clinics (including selected data on patient demographics, ART cycle and cycle outcomes) are compared with information recorded in medical records and discrepancy rates are calculated. The discrepancy rate for 
pregnancy outcome has consistently been around I\% (CDC, 20I3b). In 201 I, 45 I clinics reported $>152000$ ART cycles.

ART is defined as procedures in which oocytes and sperm or embryos are handled outside the patient's body, such as in vitro fertilization (IVF). IVF involves extracting a woman's eggs, fertilizing the eggs in the laboratory, and then transferring the resulting embryo(s) into the woman's uterus. The embryos can be transferred either immediately following fertilization and culture (fresh embryo transfer cycles), or frozen and used at a later time (frozen/thawed embryo transfer cycles). The definition of ART also includes rare procedures when gametes or zygotes are transferred into fallopian tubes (gamete intrafallopian transfer [GIFT] and zygote intrafallopian transfer $[\mathrm{ZIFT}]$, respectively). All variables related to current or previous ART procedures were extracted from NASS. Infertility diagnoses were not mutually exclusive and included tubal factor, ovulation disorders, diminished ovarian reserve, endometriosis, uterine factor, male factor, other causes and unexplained causes. Intracytoplasmic sperm injection (ICSI) is a procedure in which a single sperm is injected into the oocyte. The collection of semen for ICSI can be done by either surgical extraction (epididymal aspiration or testicular biopsy) or non-surgical method (ejaculation). Embryo stage at transfer was calculated by subtracting the oocyte retrieval date from the embryo transfer date. Typically, embryos transferred on Day 3 correspond to the cleavage stage of development, whereas embryos transferred on Day 5 correspond to the blastocyst stage. Assisted hatching was defined as the purposeful disruption of an embryo's zona pellucida by laser, mechanical or chemical means in an effort to improve implantation rates among poor prognosis patients or on embryos noted to have a thick zona pellucida.

\section{Outcome (autism and related variables)}

The administrative Autism Caseload database is maintained by the California DDS, the agency through which the State of California provides services and support to individuals with developmental disabilities, and includes information on the vast majority of persons with autism in California (Croen et al., 2002; DDS, 2002; Sunderam et al., 2013). Since inclusion in the database is based on the eligibility for services, it includes cases with a higher level of functional deficit, such as autistic disorder (as opposed to pervasive developmental disorder not otherwise specified or Asperger's disorder), within the commonly accepted category of autism spectrum disorders (ASD).

Autistic disorder is defined by the American Psychiatric Association's Diagnostic and Statistical Manual, 4th edition (DSM-IV, 1994, revised in 2000), which provides standards for the diagnosis and classification of ASD (APA, 2000). The DDS Autism Caseload database includes children diagnosed under the DSM-IV code 299.0, which includes autistic disorder (hereafter, autism). All variables related to autism diagnosis were extracted from DDS Autism Caseload database. The date of autism diagnosis was defined as the date of enrollment on the DDS Autism Caseload database. Since the majority of autism cases are typically diagnosed by age 5 ( $85.3 \%$ in our sample), we allowed 5 years of follow-up for each child in the study.

We used information from the Client Development Evaluation Report (CDER) database of the DDS to evaluate symptom severity and functioning at the child's initial DDS evaluation. The CDER uses three items to measure communication (word usage, receptive language and expressive language) and five items to measure social functioning (interaction with peers and nonpeers, friendship formation and maintenance, and participation in social activities). We created equally weighted communication and social functioning indices by summing the scores for each element in a given construct and dividing by the total number of elements. The resulting scores were then standardized by age at diagnosis and birth year; communication and social index scores in the bottom tertile were defined as low functioning. Due to changes in the evaluation criteria after 2008 , we were only able to assess symptom severity for children born during 1997-2004.

\section{Other variables}

Variables extracted from California Birth Certificate include: maternal and paternal age at birth, maternal education, maternal race and ethnicity, number of previous births, mode of delivery, infant sex, plurality, gestation age, infant birthweight, and birth year.

\section{Statistical analysis}

All analyses were conducted using SUDAAN software (Version 10.0, Research Triangle Institute, Research Triangle Park, NC). Since almost half of ART-conceived infants are born in multiple gestations, and due to possible correlation of the outcomes of siblings from the same gestation, we accounted for cluster effect by specifying delivery as a cluster with one or more observations (Hibbs et al., 20I0).

We first calculated trends of the incidence of autism diagnosis within the first 5 years of life among ART-conceived singletons and multiples born in California between 1997 and 2006 and calculated $P$-values for trend.

We then calculated percent distribution of selected infant, parental, and treatment characteristics by autism diagnosis. We compared percent distribution of these characteristics between children diagnosed with autism and those not diagnosed using chi-square test and reported $P$-values.

We then selected characteristics that were significant at $P<0.05$ level for inclusion in Cox proportional hazards models (one for singletons and one for multiples) to explore the association of autism diagnosis with these characteristics. Male factor infertility and birth year were included in the models on the basis of a priori decision. Because of collinearity between number of fetal hearts at 6-week ultrasound and number of embryos transferred, only the former variable was included in the models. We estimated crude, and through Cox proportional hazards models, adjusted, hazard risk ratios (HRR) and $95 \%$ confidence intervals (Cl); due to a high percentage $(97 \%)$ of missing ICSI data for frozen/thawed embryo transfer cycles (represented $15 \%$ of all cycles), we used a weight-adjusted approach (SUDAAN's WTADJUST procedure) to adjust for missing values. We report unadjusted and adjusted HRRs for our primary exposures of interest, ART treatment factors and parental infertility diagnoses. Infant and parental characteristics that were adjusted for included infant sex (male, female), gestational age ( $\geq 37$ weeks, $<37$ weeks), birthweight ( $\geq 2500 \mathrm{~g},<2500 \mathrm{~g}$ ), maternal and paternal age at delivery ( $<35$ years, $35-39$ years, $\geq 40$ years), number of previous births $(0, \geq I)$, mode of delivery (vaginal, Cesarean), and birth year. For model checking purposes, we repeated the analysis restricting the sample to fresh embryo transfer cycles without using weight-adjusted approach, and restricting multiple births to twin births only.

In additional analysis we built the models (separately for singletons and multiples) by subsequently adding variables/groups of variables to assess changes in the HRR for the association between ICSI and autism diagnosis: Model I included infant sex; Model 2 included variables in Model I, gestational age, birthweight, number of fetal hearts on 6-week ultrasound and birth year; Model 3 included variables in Model 2, maternal and paternal age at delivery, and number of previous births; Model 4 included variables in Model 3 and mode of delivery; Model 5 included variables in Model 4, male factor infertility (yes, no), tubal factor infertility (yes, no), unexplained infertility (yes, no), source of oocytes (non-donor, donor) and number of fetal hearts on 6-week ultrasound (I, 2, $\geq 3$ for singletons; $2, \geq 3$ for multiples).

Additionally, we estimated adjusted HRRs and $95 \%$ confidence intervals for the association of autism diagnosis with ICSI stratified by male factor infertility (yes, no) and method of semen collection (surgical, non-surgical) among fresh embryo transfer cycles; infants born as a result of conventional IVF without use of ICSI served as a reference group.

Finally, we assessed autism severity indicators by ICSI use among ARTconceived children diagnosed with autism. We calculated proportion of autistic children with low communication index, low social functioning 
index and co-occurring intellectual disability stratified by ICSI use and calculated risk ratios and $95 \% \mathrm{Cl}$ for the association of autism severity with ICSI.

To protect sensitive information, the study protocol prohibits publication of small cell tabulations. Therefore, the numbers $<30$ are not shown, and supplementary suppression is performed where appropriate to prevent back-calculation of small numbers.

This protocol was approved by the Institutional Review Boards at CDC and Columbia University and the California Committee for the Protection of Human Subjects.

\section{Results}

The annual incidence of autism diagnosis among ART-conceived infants born between 1997 and 2006 ranged from 0.6 to I.0\% among singletons and from 1.1 to $1.4 \%$ among multiples ( $P$ for trend 0.19 and 0.93 , respectively) (Fig. I).

Among 42383 ART-conceived infants, about half were male, over half were born in multiple gestations (47\% twin and $7 \%$ triplet or higher order gestations), $38 \%$ were born preterm and $35 \%$ had birthweight $<2500 \mathrm{~g}$ (Table I). The majority of mothers of ART-conceived infants were non-Hispanic white, college graduates, and had Cesarean delivery. Over $40 \%$ of mothers had previous ART procedures and almost $50 \%$ had previous births. Among parents of ART-conceived children, $28 \%$ of mothers and $42 \%$ of fathers were 40 years or older at time of delivery. Male factor infertility was the most common diagnosis of ART patients (37\%), followed by diminished ovarian reserve (21\%) and tubal factor infertility (20\%). ICSI was used in $52 \%$ of ART procedures; the proportion of ART cycles with ICSI increased from $33 \%$ in 1997 to $60 \%$ in 2006 . The majority of procedures utilized patient's own oocytes (79\%), freshly fertilized embryos (85\%), cleavage stage embryos (76\%) and two or more embryos (75\%). Extra embryos were cryopreserved in $52 \%$ of fresh embryo transfer cycles, and assisted hatching was used in $42 \%$ of procedures. Among all ART-conceived infants, 437 (I.0\%) were diagnosed with autism within the first 5 years of life. As compared with ART-conceived children not diagnosed with autism, a greater percentage of those diagnosed with autism were male, twins or triplets or higher order multiples, born preterm, and had low birthweight. Parents of autistic children were older than parents of children not diagnosed with autism. Compared with mothers of children without autism, mothers of autistic children were more likely to have no

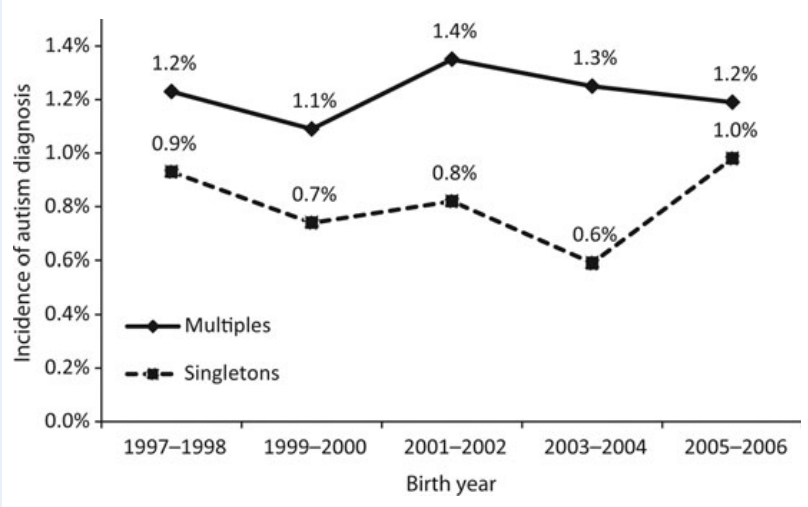

Figure I Trends of the incidence of autism diagnosis within the first 5 years of life among assisted reproductive technology-conceived children, by plurality, California, 1997-2006. prior births and have Cesarean delivery, and less likely to have tubal factor or unexplained infertility. Autism diagnosis among ART-conceived children was more prevalent with the use of ICSI, donor oocytes and transfer of $>2$ embryos.

Among ART-conceived singletons, the incidence of autism diagnosis was $0.8 \%(0.7-0.9 \%)$ (Table II). Offspring of patients with unexplained infertility had lower incidence of autism diagnosis than offspring of patients with other infertility diagnoses (adjusted HRR, 95\% Cl: 0.38 , 0.15-0.94). Use of ICSI was associated with higher incidence of autism diagnosis (adjusted HRR, 95\% Cl: 1.65, I.08-2.52). Among ART-conceived multiples, the incidence of autism diagnosis was $1.2 \%$ ( I.I-I.4\%). Offspring of patients with tubal factor infertility had lower incidence of autism diagnosis than offspring of patients with other infertility diagnoses (adjusted HRR, 95\% Cl: 0.56, 0.35-0.90). As with singletons, use of ICSI was associated with higher incidence of autism diagnosis (adjusted HRR, 95\% Cl: I.7I, I.I0-2.66). The association between ICSI and autism did not substantially change when we restricted our sample to fresh embryo transfer cycles without using weightadjusted approach (adjusted HRR, 95\% Cl: I.6I, I.05-2.46 for singletons and $1.48,1.04-2.10$ for multiples), or restricted multiple births to twin births only (adjusted HRR, 95\% Cl: 1.99, I.19-3.31).

When variables/groups of variables were added to the model in sequence, point estimates of the adjusted HRR for the association between ICSI and autism remained statistically significant, ranging from I.59 to I.66 among singletons and from I.59 to I.7I among multiples (Fig. 2).

When the ICSI group was stratified by male factor infertility and semen collection method among fresh embryo transfer cycles, the association of ICSI use with autism diagnosis was attenuated due to reduced sample, especially when ICSI was used with male factor infertility or surgical semen collection compared with conventional IVF cycles (adjusted HRR, 95\% Cl: I.23, 0.92- I.64 for ICSI with male factor infertility and I.22, 0.65-2.3I for ICSI with surgical semen collection) (Fig. 3). The association, however, remained significant when ICSI was used without male factor infertility or non-surgical semen collection (adjusted HRR, $95 \% \mathrm{Cl}: 1.57,1.18-2.09$ for ICSI without male factor infertility and I.4I, I.09-1.8I for ICSI with non-surgical semen collection).

In the analysis of autism severity indicators among a smaller sample of ART-conceived children diagnosed with autism, no statistically significant differences were observed by ICSI use status (Table III).

\section{Discussion}

Our study used one of the largest population-based datasets on ART and is the first study based in the USA to our knowledge to explore the association between ART treatment and parental infertility diagnosis with autism in ART-conceived children. We found that among ART-conceived children born in California between 1997 and 2006, the incidence of autism diagnosis remained at $\sim 0.8 \%$ among singletons and $\sim 1.2 \%$ among multiples. Incidence of autism diagnosis was higher when ICSI was used compared with conventional IVF and lower when parents had unexplained infertility (among singletons) or tubal factor infertility (among multiples) compared with other types of infertility.

There has been a significant increase in the reported prevalence of autism spectrum disorders in California, overall in the USA and in other countries (Croen et al., 2002; Yeargin-Allsopp et al., 2003; CDC, 20I3c, 20I4). During the study period, from 1997 to 2006, the 
Table I Characteristics of assisted reproductive technology (ART)-conceived children and related ART procedures, and autism diagnosis within the first 5 years of life, California, 1997-2006.

\begin{tabular}{|c|c|c|c|c|}
\hline Characteristics $^{\mathrm{a}}$ & $\begin{array}{l}\text { All ART-conceived } \\
\text { infants } \\
\%(n)\end{array}$ & $\begin{array}{l}\text { Infants diagnosed } \\
\text { with autism } \\
\%(n)\end{array}$ & $\begin{array}{l}\text { Infants not diagnosed } \\
\text { with autism } \\
\%(n)\end{array}$ & $P$-value \\
\hline \multicolumn{5}{|l|}{ Infant characteristics } \\
\hline Infant sex & & & & $<0.0001$ \\
\hline Male & $50.8(2 \mid 548)$ & $78.7(344)$ & $50.6(21204)$ & \\
\hline Female & $49.2(20835)$ & $21.3(93)$ & $49.4(20742)$ & \\
\hline Plurality & & & & $<0.000$ I \\
\hline Singleton & $46.7(19790)$ & $36.4(159)$ & $46.8(19631)$ & \\
\hline Twin & $46.7(19772)$ & $50.3(220)$ & $46.6(19552)$ & \\
\hline Triplet and higher order multiple & $6.7(282 \mathrm{I})$ & $13.3(58)$ & $6.6(2763)$ & \\
\hline Gestational age & & & & $<0.0001$ \\
\hline$\geq 37$ weeks & $61.8(26176)$ & $48.3(211)$ & $61.9(25965)$ & \\
\hline$<37$ weeks & $38.2(16199)$ & $51.7(226)$ & 38.1 ( 15973$)$ & \\
\hline Birthweight & & & & $<0.0001$ \\
\hline$\geq 2500 \mathrm{~g}$ & $65.4(27703)$ & $54.2(237)$ & $65.5(27466)$ & \\
\hline$<2500 \mathrm{~g}$ & $34.6(14652)$ & $45.8(200)$ & $34.5(14452)$ & \\
\hline Number of fetal hearts on 6-week ultrasound & & & & $<0.0001$ \\
\hline I fetal heart & $43.1(18161)$ & $32.3(139)$ & $43.1(18022)$ & \\
\hline 2 fetal hearts & $42.1(17767)$ & $43.9(189)$ & $42.1(17578)$ & \\
\hline$\geq 3$ fetal hearts & $14.8(6247)$ & $23.4(101)$ & $\mid 4.7(6 \mid 46)$ & \\
\hline Birth year & & & & 0.66 \\
\hline $1997-1998$ & II.I (4702) & $11.9(52)$ & II.I (4650) & \\
\hline 1999-2000 & $14.2(6049)$ & $12.8(56)$ & I 4.3 (5993) & \\
\hline $200 I-2002$ & $20.9(8836)$ & $22.4(98)$ & $20.8(8738)$ & \\
\hline $2003-2004$ & $26.0(11013)$ & $23.6(103)$ & $26.0(10910)$ & \\
\hline $2005-2006$ & $27.8(1 \mid 783)$ & $29.3(128)$ & $27.8(11655)$ & \\
\hline \multicolumn{5}{|l|}{ Parental characteristics } \\
\hline Maternal age at delivery & & & & 0.04 \\
\hline$<35$ years & $34.6(14657)$ & $32.0(140)$ & $34.6(14517)$ & \\
\hline $35-39$ years & $37.6(15923)$ & $33.9(148)$ & $37.6(15775)$ & \\
\hline$\geq 40$ years & $27.8(|| \mid 803)$ & $34.1(149)$ & $27.8(1 \mid 654)$ & \\
\hline Maternal race/ethnicity & & & & 0.22 \\
\hline Non-Hispanic white & $70.3(29 \mid 36)$ & $66.6(285)$ & $70.4(28851)$ & \\
\hline Non-Hispanic black & $2.4(1001)$ & Not shown ${ }^{\mathrm{b}}$ & Not shown ${ }^{\mathrm{b}}$ & \\
\hline Hispanic & II.I (4607) & Not shown ${ }^{\mathrm{b}}$ & Not shown ${ }^{\mathrm{b}}$ & \\
\hline Asian/Pacific Islander & I6.I (668I) & I6.I (69) & $16.1(66 \mid 2)$ & \\
\hline Maternal education & & & & 0.06 \\
\hline$\leq$ High school graduate & I2.0 (4924) & $9.3(39)$ & $12.0(4885)$ & \\
\hline Some college & I8.I (7446) & $22.1(93)$ & I8.| (7353) & \\
\hline College graduate & $69.9(28778)$ & $68.6(288)$ & $70.0(28490)$ & \\
\hline Paternal age & & & & 0.02 \\
\hline$<35$ years & $24.2(10057)$ & $22.0(94)$ & $24.2(9963)$ & \\
\hline $35-39$ years & $33.8(14067)$ & $28.7(123)$ & 33.9 (13 944) & \\
\hline$\geq 40$ years & $42.0(17434)$ & $49.3(211)$ & $41.9(17223)$ & \\
\hline
\end{tabular}


Table I Continued

\section{Characteristics $^{a}$}

Number of previous ART procedures

0

$\geq 1$

Unknown/missing

Number of previous births

0

$\geq 1$

Mode of delivery

Vaginal delivery

Cesarean delivery

Infertility diagnosis and ART treatment characteristics

Infertility diagnosis ${ }^{c}$

Tubal factor

$$
\text { Yes }
$$

$$
\text { No }
$$

Ovulation disorders

Yes

No

Diminished ovarian reserve

Yes

No

Endometriosis

Yes

No

Uterine factor

Yes

No

Male factor

Yes

No

Other causes

Yes

No

Unexplained causes

Yes

No

Method of embryo fertilization

Conventional IVF (without ICSI)

ICSI

ICSI unknown ${ }^{d}$

Source of oocytes

Non-donor (patient's) oocytes

Donor oocytes

\section{All ART-conceived infants \\ $\%(n)$}

$43.6(18468)$

40.4 (17 129)

$16.0(6786)$

$50.6(2 \mid 442)$

$49.4(20912)$

$35.9(15231)$

$64.1(27$ I52)

78.8 (33 399)

21.2 (8969)

Infants diagnosed
with autism
$\%(n)$
with autism

Infants not diagnosed with autism ${ }^{b}$

$\%(n)$

$39.6(173)$

$44.2(193)$

$16.2(7 \mathrm{I})$

55.7 (243)

$44.3(193)$

$24.9(109)$

75.1 (328)

$14.8(63)$

$85.2(363)$

10.8 (46)

$89.2(380)$

$24.4(104)$

$75.6(322)$

12.7 (54)

$87.3(372)$

7.0 (30)

$93.0(396)$

$37.6(160)$

$62.4(266)$

16.7 (7I)

83.3 (355)

$7.0(30)$

$93.0(396)$

$27.1(117)$

60.0 (259)

$13.0(56)$

$72.8(318)$

$27.2(119)$
50.6 (21 199)

49.4 (20719)

36. I (15 122)

63.9 (26 824)

19.6 (8082)

80.4 (33 I54)

9. I (3762)

$90.9(37474)$

20.5 (8455)

79.5 (32 78I)

$12.2(5050)$

$87.8(36186)$

4.8 (1987)

95.2 (39 249)

37.0 (15 267)

63.0 (25 969)

15.9 (6554)

84.I (34 682)

10.9 (4499)

89.1 (36 737)

$33.1(13636)$

52.1 (2। 469)

$14.8(6 \mid 17)$

$78.9(3308 \mathrm{I})$

2I.I (8850)
0.03

0.10

0.01

0.32

0.09

0.81

0.10

0.01

P-value

$<0.000$ ।

0.83

0.70

$<0.0$ I

$<0.0$ I

Continued 
Table I Continued

$\begin{array}{ll}\text { Characteristics }^{\mathrm{a}} & \begin{array}{l}\text { All ART-conceived } \\ \text { infants } \\ \%(n)\end{array}\end{array}$

Infants diagnosed
with autism
$\%(n)$

Type of embryos used

Fresh embryos

Frozen/thawed embryos

Days of embryo culture (for fresh ET only) ${ }^{\mathrm{e}}$

3 days
5 days

Extra embryos cryopreserved (for fresh ET only)

Yes

No

Number of embryos transferred

$\leq 2$ embryos
3 embryos
4 embryos
$\geq 5$ embryos

Assisted hatching

Yes

No
$85.0(35996)$

$15.0(6372)$

$75.9(21796)$

13.4 (3836)

$52.3(18795)$

47.7 (17 163)

$25.5(10816)$

$31.3(13271)$

$24.0(10147)$

$19.2(8 \mid 27)$

41.8 (I 7521$)$

$58.2(24388)$
$87.2(38 I)$

$12.8(56)$

$77.8(217)$

$12.5(35)$

$51.2(195)$

48.8 (186)

20.1 (88)

31.8 (139)

$19.0(83)$

\section{Infants not diagnosed with autism ${ }^{\mathrm{b}}$ \\ $\%$ (n)}

$P$-value

0.20

$84.9(35615)$

I5.I (6316)

0.96

75.9 (2। 579)

I3.4 (380I)

0.69

$52.3(18600)$

47.7 (16977)

0.04

$25.6(10728)$

$31.3(13$ 132)

$23.9(10020)$

19.2 (8044)

0.59

$41.8(17336)$

$58.2(24$ । 45$)$

ET, embryo transfer.

a'Unknown/missing' category is shown if $>3 \%$ of values are unknown or missing; otherwise, these values are excluded from the calculations.

bData not shown due to confidentiality requirements to suppress small cell tabulations; supplementary suppression is performed where appropriate.

Infertility diagnoses are not mutually exclusive.

d Information on ICSI use is routinely collected for fresh embryo transfer cycles (<1\% missing), but not for frozen/thawed embryo transfer cycles ( $97 \%$ missing).

eLimited to 3 and 5 days of embryo culture.

incidence of autism diagnosis among ART-conceived children did not show a similar increase. This may be explained by the positive trend in ART practice in the USA, where fewer embryos are transferred during the procedure (Steinberg et al., 2013) and more ART-conceived infants are born as term singletons with normal birthweight (Joshi et al., 2012), which have been shown to be inversely associated with autism diagnosis (Schieve et al., 20I I).

The inverse association of unexplained and tubal factor infertility with autism diagnosis has not been previously described and might be related to the characteristics of patients. Patients with unexplained infertility (I4\% of all ART cycles performed in the USA in 20II (CDC, 20।3b)) and patients with tubal factor infertility (I5\% of all ART cycles in 2010 (Kawwass et al., 20I3)) tend to be younger. In addition, patients for whom no cause of infertility was found at the time of ART cycle may have less severe fertility problems. It is important to note that since our study was restricted to ART-conceived children, the observed associations are not relevant to infertility patients who did not have ART.

The fact that ICSI use was associated with increased incidence of autism diagnosis after accounting for infant, parental or treatment factors, suggests that the association cannot be explained by characteristics known to be associated with ICSI procedures, such as diagnosis of male factor infertility or older parental age. In fact, the associations with autism diagnosis were stronger when ICSI was used without male factor infertility and with non-surgical method of semen collection. ICSI, injection of a single sperm into an egg, was developed to improve fertilization in couples with male factor infertility, and is not recommended for routine use in couples with other infertility causes (ASRM, 2012). Nevertheless, the use of ICSI in the USA more than doubled from 30\% in 1996 (CDC, 1998) to 67\% in 20II (CDC, 20I3a) in all ART cycles, not only those for patients with male factor infertility. Interestingly, we did not observe similar increase in autism diagnosis among ART-conceived infants during the same time period, which may be explained by the simultaneous decline of other risk factors shown to be associated with autism, namely multiple births and low birthweight. Although the exact mechanism of the association between ICSI and neuro-behavioral disorders, including autism, is yet to be determined, it has been suggested that epigenetic modifications resulting from either the procedure itself or characteristics of the patients using the procedure may play a role (De Rycke et al., 2002; Paoloni-Giacobino and Chaillet, 2004; Dada et al., 2012). Although several studies reported an association between oligospermia and abnormal imprinting (Marques et al., 2004, 2008; Dasoula et al., 2007; Kobayashi et al., 2007), our results suggest that male factor infertility may not be the main risk factor for autism diagnosis in offspring. The association of ICSI use with no male factor infertility or non-surgical semen collection with autism diagnosis contradicts the recent study from Sweden which showed that fresh embryo transfers involving ICSI with surgically-extracted sperm (indicator of male factor infertility) were associated with autistic disorder, compared with transfers not involving ICSI (Sandin et al., 20I3), and needs to be investigated further. Possible explanations include the use of ICSI as a rescue technique when fertilization with conventional IVF fails among patients without male factor infertility (Beck-Fruchter et al., 20l4), or 
Table II Association of autism diagnosis within the first 5 years of life with selected infertility diagnoses and assisted reproductive technology (ART) treatment characteristics among ART-conceived children, by plurality, California, 1997-2006.

\begin{tabular}{|c|c|c|c|}
\hline Characteristics & $\begin{array}{l}\text { Incidence of autism diagnosis } \\
\%(95 \% \mathrm{Cl})\end{array}$ & $\begin{array}{l}\text { Crude hazard risk } \\
\text { ratio }(95 \% \mathrm{Cl})\end{array}$ & $\begin{array}{l}\text { Adjusted' hazard risk } \\
\text { ratio }(95 \% \mathrm{Cl})\end{array}$ \\
\hline \multicolumn{4}{|l|}{ Singletons } \\
\hline Overall & $0.8(0.7-0.9)$ & - & - \\
\hline \multicolumn{4}{|l|}{ Infertility diagnosis } \\
\hline \multicolumn{4}{|l|}{ Tubal factor } \\
\hline Yes & $0.7(0.5-1.1)$ & $0.88(0.58-1.33)$ & $0.89(0.54-1.48)$ \\
\hline No & $0.8(0.7-1.0)$ & Reference & Reference \\
\hline \multicolumn{4}{|l|}{ Unexplained causes } \\
\hline Yes & $0.4(0.2-0.8)$ & $0.5 I(0.26-1.01)$ & $0.38(0.15-0.94)$ \\
\hline No & $0.8(0.7-1.0)$ & Reference & Reference \\
\hline \multicolumn{4}{|l|}{ Male factor } \\
\hline Yes & $0.9(0.7-1.1)$ & I.II (0.81-1.52) & $0.89(0.60-1.32)$ \\
\hline No & $0.8(0.6-0.9)$ & Reference & Reference \\
\hline \multicolumn{4}{|l|}{ Method of embryo fertilization } \\
\hline ICSI & $0.9(0.8-1.1)$ & $1.55(1.05-2.29)$ & $1.65(1.08-2.52)$ \\
\hline Conventional IVF (without ICSI) & $0.6(0.4-0.8)$ & Reference & Reference \\
\hline \multicolumn{4}{|l|}{ Source of oocytes } \\
\hline Donor oocytes & $1.0(0.7-1.4)$ & $1.30(0.90-1.87)$ & $1.08(0.68-|.7|)$ \\
\hline Non-donor (patient's) oocytes & $0.8(0.6-0.9)$ & Reference & Reference \\
\hline \multicolumn{4}{|l|}{ Fetal hearts on 6-week ultrasound } \\
\hline I fetal heart & $0.8(0.7-0.9)$ & Reference & Reference \\
\hline 2 fetal hearts & $0.9(0.5-1.6)$ & $1.20(0.66-2.15)$ & $1.17(0.61-2.27)$ \\
\hline$\geq 3$ fetal hearts & $1.6(0.7-3.8)$ & $2.10(0.86-5.11)$ & $2.34(0.91-5.97)$ \\
\hline \multicolumn{4}{|l|}{ Multiples } \\
\hline Overall & $1.2(1.1-1.4)$ & - & - \\
\hline \multicolumn{4}{|l|}{ Infertility diagnosis } \\
\hline \multicolumn{4}{|l|}{ Tubal factor } \\
\hline Yes & $0.8(0.6-1.1)$ & $0.61(0.43-0.87)$ & $0.56(0.35-0.90)$ \\
\hline No & $1.3(1.2-1.5)$ & Reference & Reference \\
\hline \multicolumn{4}{|l|}{ Unexplained causes } \\
\hline Yes & $0.9(0.5-1.4)$ & $0.67(0.4 I-1.11)$ & $0.62(0.32-1.23)$ \\
\hline No & $1.3(1.1-1.5)$ & Reference & Reference \\
\hline \multicolumn{4}{|l|}{ Male factor } \\
\hline Yes & $1.2(1.0-1.5)$ & $0.97(0.73-1.27)$ & $0.70(0.44-1.14)$ \\
\hline No & $1.2(1.1-1.5)$ & Reference & Reference \\
\hline \multicolumn{4}{|l|}{ Method of embryo fertilization } \\
\hline ICSI & $1.4(1.1-1.8)$ & $1.54(1.09-2.18)$ & $1.71(1.10-2.66)$ \\
\hline Conventional IVF (without ICSI) & $0.9(0.7-1.2)$ & Reference & Reference \\
\hline \multicolumn{4}{|l|}{ Source of oocytes } \\
\hline Donor oocytes & $1.6(1.2-2.0)$ & $1.40(1.04-1.88)$ & $0.85(0.53-1.36)$ \\
\hline Non-donor (patient's) oocytes & I.I (I.0- I.3) & Reference & Reference \\
\hline \multicolumn{4}{|l|}{ Fetal hearts on 6-week ultrasound } \\
\hline 2 fetal hearts & I.I $(0.9-1.3)$ & Reference & Reference \\
\hline$\geq 3$ fetal hearts & $1.6(1.3-2.1)$ & $1.51(1.13-2.02)$ & $1.12(0.80-1.56)$ \\
\hline
\end{tabular}

$\mathrm{Cl}$, confidence interval.

'Adjusted for infant sex (male, female), gestational age ( $\geq 37$ weeks, $<37$ weeks), birthweight ( $\geq 2500 \mathrm{~g},<2500$ g), maternal and paternal age at delivery $(<35$ years, $35-39$ years, $\geq 40$ years), number of previous births $(0, \geq 1)$, mode of delivery (vaginal, Cesarean), and birth year. 

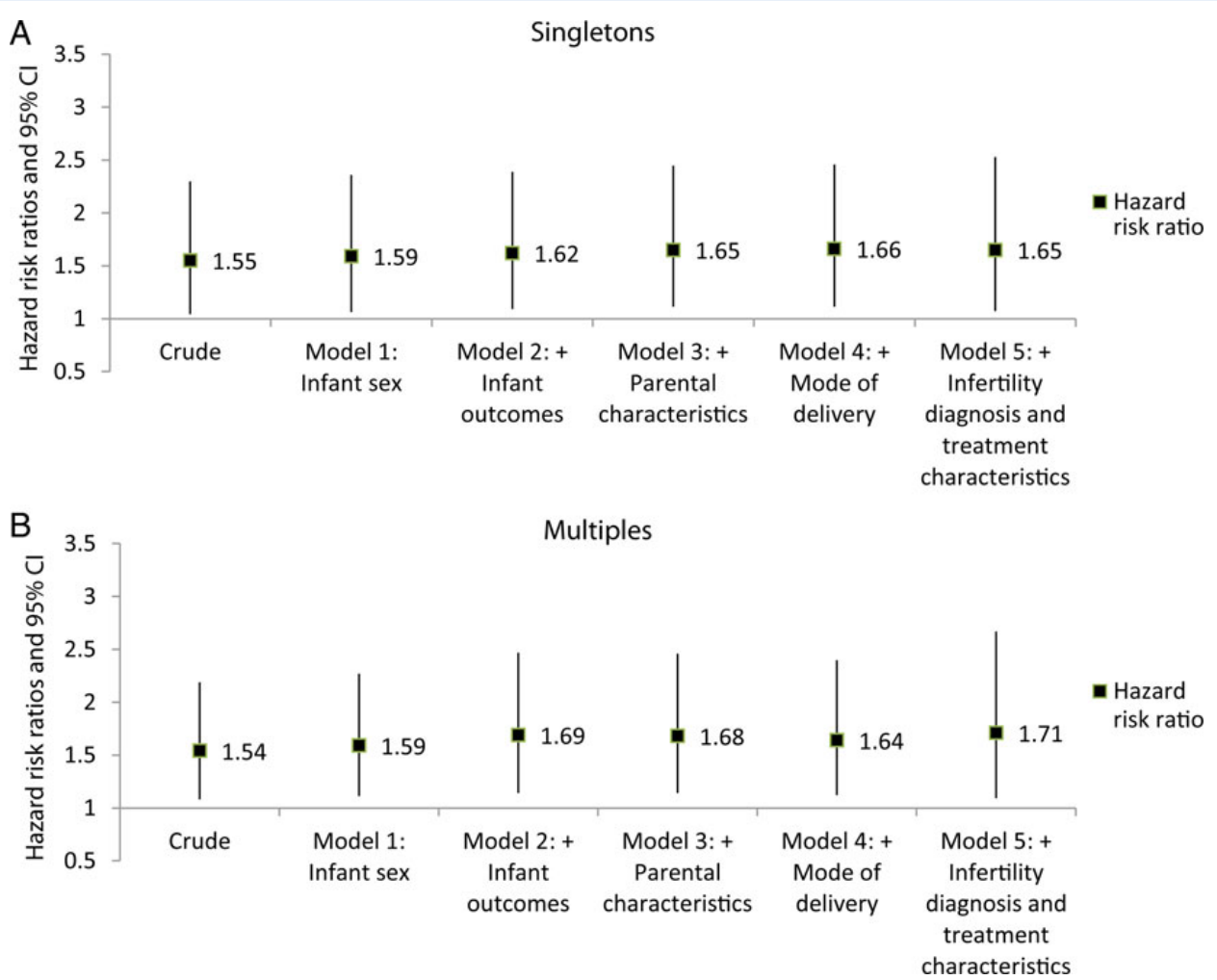

Figure 2 Crude and adjusted hazard risk ratios and $95 \%$ confidence intervals $(\mathrm{Cl})$ for the association of autism diagnosis within the first 5 years of life with ICSI among assisted reproductive technology-conceived singletons (A) and multiple (B), California, 1997-2006. The following adjustments were used: Model I: infant sex (male, female); Model 2: variables in Model I + gestational age ( $\geq 37$ weeks, $<37$ weeks), birthweight $(\geq 2500 \mathrm{~g},<2500 \mathrm{~g})$, number of fetal hearts on 6-week ultrasound ( $1,2, \geq 3$ for singletons; $2, \geq 3$ for multiples), birth year; Model 3: variables in Model $2+$ maternal and paternal age at delivery ( $<35$ years, $35-39$ years, $\geq 40$ years), number of previous births $(0, \geq 1)$; Model 4: variables in Model $3+$ mode of delivery (vaginal, Cesarean); Model 5: variables in Model 4 + male factor infertility (yes, no), tubal factor infertility (yes, no), unexplained infertility (yes, no), source of oocytes (non-donor, donor). Reference group: infants born after conventional IVF (without ICSI).

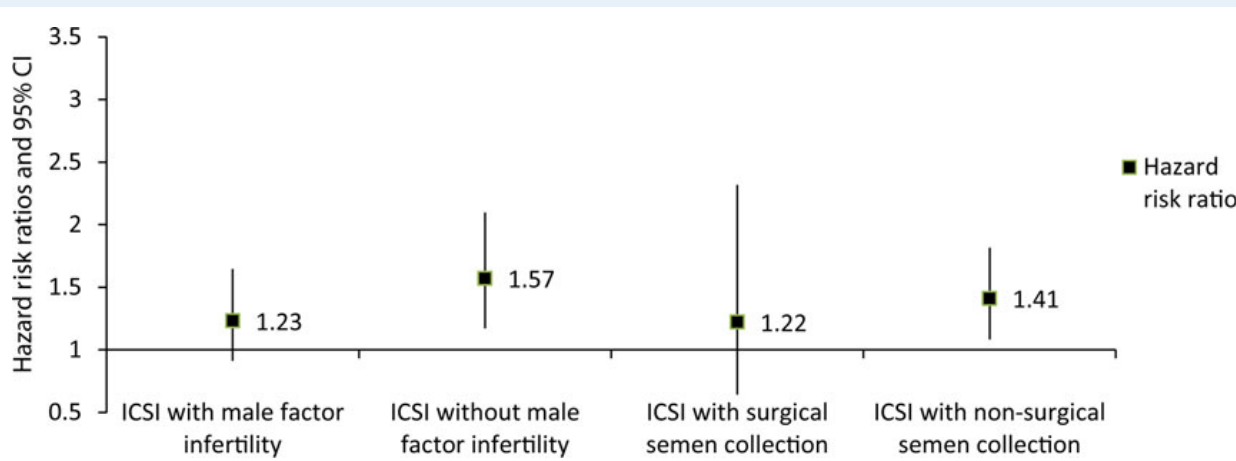

Figure 3 Adjusted hazard risk ratios and $95 \%$ confidence intervals $(\mathrm{Cl})$ for the association of autism diagnosis within the first 5 years of life with ICSI among assisted reproductive technology-conceived children, by male factor infertility and method of semen collection, fresh embryo transfer cycles, California, 1997-2006. The following adjustments were used: infant sex (male, female), gestational age ( $\geq 37$ weeks, $<37$ weeks), birthweight ( $\geq 2500$ g, $<2500 \mathrm{~g}$ ), maternal and paternal age at delivery ( $<35$ years, $35-39$ years, $\geq 40$ years), number of previous births $(0, \geq \mathrm{l})$, mode of delivery (vaginal, Cesarean), birth year, tubal factor infertility (yes, no), unexplained infertility (yes, no), source of oocytes (non-donor, donor), fetal hearts on 6-week ultrasound $(I, 2, \geq 3)$, and plurality (singleton, multiple). Reference group: infants born after conventional IVF (without ICSI).

under-reporting/under-diagnosis of male factor infertility. Our study provides data on autism severity by ICSI use, although no differences in the distribution of these factors were statistically significant, possibly due to small sample size. In our study, co-occurrence of intellectual disability with autism among ART-conceived children (19.I and II.2\% for ICSI and conventional IVF, respectively) is lower than previously 


\begin{tabular}{|c|c|c|c|}
\hline Severity indicators & ICSI, \% & $\begin{array}{l}\text { Conventional } \\
\text { IVF, \% }\end{array}$ & $\begin{array}{l}\text { Risk ratio } \\
(95 \% \mathrm{Cl})\end{array}$ \\
\hline $\begin{array}{l}\text { Low communication } \\
\text { index }(n=258)\end{array}$ & 18.3 & 24.5 & $\begin{array}{l}0.75 \\
(0.44-1.28)\end{array}$ \\
\hline $\begin{array}{l}\text { Low social functioning } \\
\text { index }(n=258)\end{array}$ & 29.9 & 21.3 & $\begin{array}{l}1.40 \\
(0.87-2.27)\end{array}$ \\
\hline $\begin{array}{l}\text { Low social functioning or } \\
\text { communication index } \\
(n=258)\end{array}$ & 39.6 & 37.2 & $\begin{array}{l}1.06 \\
(0.75-1.5 I)\end{array}$ \\
\hline $\begin{array}{l}\text { Co-occurring intellectual } \\
\text { disability }(n=27 \mathrm{I})\end{array}$ & 19.1 & 11.2 & $\begin{array}{l}1.70 \\
(0.79-3.66)\end{array}$ \\
\hline
\end{tabular}

reported among all children with autism (regardless of conception method), although there is wide variability by state (CDC, 2014). It has been reported that prevalence of intellectual disability in autistic children is lower when they come from households with higher income (Rivard et al., 20I4), which is the case for ART-conceived children in the USA.

The results of the study should be considered in light of several limitations. Linkages between National ART Surveillance System data, Birth Certificate data and Autism Caseload data are imperfect, although acceptable for surveillance purposes ( $\sim 90 \%$ linkage rates). Unlinked records likely result from ART procedures conducted out of state or migration to California after birth of a child in another state. According to the American Community Survey, $\sim 7 \%$ of children under the age of 5 years living in California in 2005-2007 were born outside of the state of residence (Census, 2005-2007). Another limitation is that enrollment in the California Department of Developmental Services is voluntary and may not represent all children diagnosed with autism in the state. Some cases of autism may be either undiagnosed or underrepresented due to limited access to the DDS system, which may underestimate the incidence of autism. Although we lacked the data on embryo quality, we used cryopreservation of extra embryos, which has been demonstrated to be a good predictor of embryo quality (Stern et al., 2012). Since we used multiple possible predictors of autism diagnosis and a number of models, some of our results can be due to chance alone. Limitations of the observational study design could affect our analysis: although we controlled for several important patient and treatment characteristics, we cannot exclude the possibility of residual confounders. It is also important to note that information about ICSI use was missing for most frozen/ thawed embryo transfer cycles, and therefore our findings of association of ICSI use and autism diagnosis can only be generalizable to fresh embryo transfer cycles.

One of the study strengths includes large sample size with detailed information on ART cycle allowing adjustment for important confounders. Restriction to ART population minimized the effect of the underlying causes of infertility and access to treatment on the outcome of interest, which is a common limitation of studies comparing ART and non-ART populations. In addition, our study contributes to the literature by investigating the population of ART-conceived children in the USA, whose circumstances of conception are different from previously studied populations of ART-conceived children outside of the USA, primarily in Scandinavia. For example, the use of blastocyst transfer, assisted hatching, ICSI and transfer of $>$ I embryo is generally higher in the USA than in Europe (Chambers et al., 2009; Baker et al., 20 I0; Sullivan et al., 20 I3).

Our study provides additional evidence of the association between some types of ART procedures with autism diagnosis among ARTconceived children. Additional research is required to explain the increased risk of autism diagnosis with ICSI use. Given the widespread use of ICSI in the USA and lack of convincing evidence of its effectiveness among patients without male factor infertility (ASRM, 20I2), more studies on the effectiveness and safety of ICSI, as well as dissemination of existing evidence among ART providers are needed. Our study shows that linking national ART surveillance data with state-based outcome data, such as the one with Autism Caseload data, is a feasible and efficient way to study long-term outcomes of ART.

\section{Acknowledgements}

The findings and conclusions in this report are those of the authors and do not necessarily represent the official position of the Centers for Disease Control and Prevention.

\section{Authors' roles}

All authors contributed to study conception and design; Y.Z. and C.F. performed data linkages and analyses; all authors contributed to data analysis and/or interpretation; D.M.K. drafted the manuscript; all authors reviewed, critically revised and approved the manuscript.

\section{Funding}

This research is partially supported by the NIH Director's Pioneer Award program, part of the NIH Roadmap for Medical Research, through grant number I DPI OD003635-0I and the National Institutes of Mental Health award number R2IMH096/22. The funders had no role in study design, data collection and analysis, decision to publish, or preparation of the manuscript.

\section{Conflict of interest}

All authors declare that they have no interests that may be relevant to the submitted work.

\section{References}

Adamson GD, Tabangin M, Macaluso M, de Mouzon J. The number of babies born globally after treatment with the assisted reproductive technologies (ART). Abstract, American Society for Reproductive Medicine (ASRM), 2013.

APA. American Psychiatric Association. Diagnostic and Statistical Manual of Mental Disorders: DSM-IV, 4th ed. Washington, DC: American Psychiatric Association; 1994, revised 2000. 2000.

ASRM. American Society for Reproductive Medicine, Society for Assisted Reproductive Technology, Practice Committee. Intracytoplasmic sperm 
injection (ICSI) for non-male factor infertility: a committee opinion. Fertil Steril 2012;98:1395-1399.

Baker VL, Jones CE, Cometti B, Hoehler F, Salle B, Urbancsek J, Soules MR. Factors affecting success rates in two concurrent clinical IVF trials: an examination of potential explanations for the difference in pregnancy rates between the United States and Europe. Fertil Steril 2010; 94: |287-|29|.

Bay B, Mortensen EL, Hvidtjorn D, Kesmodel US. Fertility treatment and risk of childhood and adolescent mental disorders: register based cohort study. BMJ 20I3;347:f3978.

Beck-Fruchter R, Lavee M, Weiss A, Geslevich Y, Shalev E. Rescue intracytoplasmic sperm injection: a systematic review. Fertil Steril 2014; 101:690-698.

CDC. Centers for Disease Control and Prevention, American Society for Reproductive Medicine, Society for Assisted Reproductive Technology. 1996 Assisted Reproductive Technology Fertility Clinic Success Rates and National Summary Report. Atlanta: U.S. Department of Health and Human Services, 1998.

CDC. Centers for Disease Control and Prevention, American Society for Reproductive Medicine, Society for Assisted Reproductive Technology. 201I Assisted Reproductive Technology Fertility Clinic Success Rates Report. Atlanta: U.S. Department of Health and Human Services, 20I3a. CDC. Centers for Disease Control and Prevention, American Society for Reproductive Medicine, Society for Assisted Reproductive Technology. 20II Assisted Reproductive Technology National Summary Report. Atlanta: U.S. Department of Health and Human Services, 20I3b.

CDC. Centers for Disease Control and Prevention, National Center for Health Statistics. Changes in prevalence of parent-reported autism spectrum disorder in school-aged US children: 2007 to 2011-2012. National Health Statistics Report; 65, 2013c.

CDC. Centers for Disease Control and Prevention. Prevalence of autism spectrum disorder among children aged 8 years-Autism and Developmental Disabilities Monitoring Network, I I sites, United States, 2010. Morb Mortal Wkly Rep Surveill Summ 2014;63(Suppl 2): I -21.

Census. United States Census Bureau, 2005-2007 American Community Survey. http://factfinder2.census.gov (accessed 15 September 2014). 2005-2007.

Chambers GM, Sullivan EA, Ishihara O, Chapman MG, Adamson GD. The economic impact of assisted reproductive technology: a review of selected developed countries. Fertil Steril 2009;9 1:228I -2294.

Conti E, Mazzotti S, Calderoni S, Saviozzi I, Guzzetta A. Are children born after assisted reproductive technology at increased risk of autism spectrum disorders? A systematic review. Hum Reprod 2013 28:3316-3327.

Croen LA, Grether JK, Hoogstrate J, Selvin S. The changing prevalence of autism in California. J Autism Dev Disord 2002;32:207-2I5.

Dada R, Kumar M, Jesudasan R, Fernandez JL, Gosalvez J, Agarwal A. Epigenetics and its role in male infertility. J Assist Reprod Genet 2012; 29:213-223.

Dasoula A, Georgiou I, Kontogianni E, Sofikitis N, Syrrou M. Methylation status of the SNRPN and HUMARA genes in testicular biopsy samples. Fertil Steril 2007;87:805-809.

DDS. California Department of Developmental Services. Autistic Spectrum Disorders. Best practice, guidelines for screening, diagnosis, and assessment. Sacramento, California, 2002.

De Rycke M, Liebaers I, Van Steirteghem A. Epigenetic risks related to assisted reproductive technologies: risk analysis and epigenetic inheritance. Hum Reprod 2002; 1 7:2487-2494.

FCSRCA. Fertility Clinic Success Rate and Certification Act of 1992 (FCSRCA), Pub. L. No. 102-493 (24 October 1992), 1992.
Hart R, Norman RJ. The longer-term health outcomes for children born as a result of IVF treatment. Part II-Mental health and development outcomes. Hum Reprod Update 2013;1 9:244-250.

Hibbs AM, Black D, Palermo L, Cnaan A, Luan X, Truog WE, Walsh MC, Ballard RA. Accounting for multiple births in neonatal and perinatal trials: systematic review and case study. J Pediatr 20 I ; 1 56:202-208.

Hvidtjorn D, Schieve L, Schendel D, Jacobsson B, Svaerke C, Thorsen P. Cerebral palsy, autism spectrum disorders, and developmental delay in children born after assisted conception: a systematic review and meta-analysis. Arch Pediatr Adolesc Med 2009; 1 63:72-83.

Hvidtjorn D, Grove J, Schendel D, Schieve LA, Svaerke C, Ernst E, Thorsen P. Risk of autism spectrum disorders in children born after assisted conception: a population-based follow-up study. J Epidemiol Community Health 201 ;;65:497-502.

Joshi N, Kissin D, Anderson JE, Session D, Macaluso M, Jamieson DJ. Trends and correlates of good perinatal outcomes in assisted reproductive technology. Obstet Gynecol 20 I 2; | 20:843-85 I.

Kawwass JF, Crawford S, Kissin DM, Session DR, Boulet S, Jamieson DJ. Tubal factor infertility and perinatal risk after assisted reproductive technology. Obstet Gynecol 20 I 3; 1 21: I 263- 127 I.

Kobayashi H, Sato A, Otsu E, Hiura H, Tomatsu C, Utsunomiya T, Sasaki H, Yaegashi N, Arima T. Aberrant DNA methylation of imprinted loci in sperm from oligospermic patients. Hum Mol Genet 2007; 16:2542-255I.

Lehti $\bigvee$, Brown AS, Gissler M, Rihko M, Suominen A, Sourander A. Autism spectrum disorders in IVF children: a national case-control study in Finland. Hum Reprod 2013;28:812-818.

Lyall K, Pauls DL, Spiegelman D, Santangelo SL, Ascherio A. Fertility therapies, infertility and autism spectrum disorders in the Nurses' Health Study II. Paediatr Perinat Epidemiol 2012;26:361 -372.

Maimburg RD, Vaeth M. Do children born after assisted conception have less risk of developing infantile autism? Hum Reprod 2007;22: I 84 I - 1843.

Marques CJ, Carvalho F, Sousa M, Barros A. Genomic imprinting in disruptive spermatogenesis. Lancet 2004;363: I700- 1702.

Marques CJ, Costa P, Vaz B, Carvalho F, Fernandes S, Barros A, Sousa M. Abnormal methylation of imprinted genes in human sperm is associated with oligozoospermia. Mol Hum Reprod 2008; 14:67-74.

Mneimneh AS, Boulet SL, Sunderam S, Zhang Y, Jamieson DJ, Crawford S, McKane P, Copeland G, Mersol-Barg M, Grigorescu V et al. States Monitoring Assisted Reproductive Technology (SMART) Collaborative: data collection, linkage, dissemination, and use. J Womens Health 2013; 22:57। - 577 .

Palermo G, Joris H, Devroey P, Van Steirteghem AC. Pregnancies after intracytoplasmic injection of single spermatozoon into an oocyte. Lancet 1992;340: 17- 18.

Paoloni-Giacobino A, Chaillet JR. Genomic imprinting and assisted reproduction. Reprod Health 2004; I:6.

Rivard M, Terroux A, Mercier C, Parent-Boursier C. Indicators of intellectual disabilities in young children with autism spectrum disorders. J Autism Dev Disord 20I4. Epub ahead of print. July 29, 2014.

Sandin S, Nygren KG, lliadou A, Hultman CM, Reichenberg A. Autism and mental retardation among offspring born after in vitro fertilization. JAMA 2013;3 10:75-84

Schieve LA, Rice C, Devine O, Maenner MJ, Lee LC, Fitzgerald R, Wingate MS, Schendel D, Pettygrove S, van Naarden Braun K et al. Have secular changes in perinatal risk factors contributed to the recent autism prevalence increase? Development and application of a mathematical assessment model. Ann Epidemiol 20 I I;2 I:930-945.

Stein D, Weizman A, Ring A, Barak Y. Obstetric complications in individuals diagnosed with autism and in healthy controls. Compr Psychiatry 2006; 47:69-75. 
Steinberg ML, Boulet S, Kissin D, Warner L, Jamieson DJ. Elective single embryo transfer trends and predictors of a good perinatal outcomeUnited States, 1999 to 2010. Fertil Steril 2013;99:1937-1943.

Stern JE, Lieberman ES, Macaluso M, Racowsky C. Is cryopreservation of embryos a legitimate surrogate marker of embryo quality in studies of assisted reproductive technology conducted using national databases? Fertil Steril 2012;97:890-893.

Sullivan EA, Zegers-Hochschild F, Mansour R, Ishihara O, de Mouzon J, Nygren KG, Adamson GD. International Committee for Monitoring Assisted Reproductive Technologies (ICMART) world report: assisted reproductive technology 2004. Hum Reprod 2013;28: 1375- 1390.
Sunderam S, Kissin DM, Crawford S, Anderson JE, Folger SG, Jamieson DJ, Barfield WD, Division of Reproductive Health NCfCDP and Health Promotion CDC. Assisted reproductive technology surveillanceUnited States, 20 I 0. Morb Mortal Wkly Rep Surveill Summ 20 I3;62: I -24. Yeargin-Allsopp M, Rice C, Karapurkar T, Doernberg N, Boyle C, Murphy C. Prevalence of autism in a US metropolitan area. JAMA 2003;289:49-55.

Zachor DA, Ben Itzchak E. Assisted reproductive technology and risk for autism spectrum disorder. Res Dev Disabil 201 I;32:2950-2956.

Zhang Y, Cohen B, Macaluso M, Zhang Z, Durant T, Nannini A. Probabilistic linkage of assisted reproductive technology information with vital records, Massachusetts 1997-2000. Matern Child Health J 20 12; 16: I703- 1708. 